\title{
Long-Term Bond Strength of Two Benzalkonium Chloride-Modified Adhesive Systems to Eroded Dentin
}

\author{
Simon Flury, Adrian Lussi, and Anne Peutzfeldt \\ Department of Preventive, Restorative, and Pediatric Dentistry, School of Dental Medicine, University of Bern, \\ Freiburgstrasse 7, 3010 Bern, Switzerland
}

Correspondence should be addressed to Simon Flury; simon.flury@zmk.unibe.ch

Received 20 April 2017; Revised 15 June 2017; Accepted 6 July 2017; Published 10 August 2017

Academic Editor: Salvatore Sauro

Copyright (C) 2017 Simon Flury et al. This is an open access article distributed under the Creative Commons Attribution License, which permits unrestricted use, distribution, and reproduction in any medium, provided the original work is properly cited.

This study investigated the effect of benzalkonium chloride (BAC) modification of two adhesive systems on long-term bond strength to normal and artificially eroded dentin. A total of 128 extracted human molars were sectioned and the buccal and oral surfaces of each molar were ground until the dentin. One half was left untreated (normal dentin) while the other half underwent artificial erosion. Resin composite was bonded to the buccal or oral surface following treatment with Adper Scotchbond 1XT or OptiBond FL without or with $1 \%$ BAC incorporation. Shear bond strength (SBS) was measured after $24 \mathrm{~h}\left(100 \%\right.$ humidity, $\left.37^{\circ} \mathrm{C}\right)$ or 1 year (tap water, $\left.37^{\circ} \mathrm{C}\right)$. SBS results were statistically analyzed $(\alpha=0.05)$. SBS was significantly lower to artificially eroded dentin than to normal dentin $(p<0.001)$. Storage for 1 year had no effect on SBS to normal dentin but led to a significant decrease in SBS to artificially eroded dentin $(p<0.001)$. BAC incorporation decreased the $24 \mathrm{~h}$ SBS to normal dentin $(p=0.018)$, increased the $24 \mathrm{~h}$ SBS to eroded dentin $(p=0.001)$, and had no effect on the 1-year SBS for either substrate. Consequently, BAC incorporation did not improve bond durability.

\section{Introduction}

Studies have shown that the adhesive bond to dentin deteriorates over time [1-4], which may jeopardize the longterm durability of resin composite restorations. The main factor leading to reduction of the adhesive bond over time is hydrolysis of resin and collagen in the hybrid layer $[2,5]$. The hydrolytic stability of adhesive systems differs between the various classes available: adhesive systems that include application of a separate adhesive resin layer (i.e., threestep etch-and-rinse and two-step self-etch adhesive systems) have proved more stable than adhesive systems that do not comprise such a layer (i.e., two-step etch-and-rinse and onestep self-etch adhesive systems) [2]. Being more hydrophilic, the latter adhesive systems act as semipermeable membranes, attract water, and thus degrade faster. Another factor leading to degradation of resin-dentin bonds is collagenolytic activity by matrix metalloproteinases (MMPs) in dentin. MMPs are endogenous enzymes that are released and activated when exposed to an acidic environment such as the one created by etching with phosphoric acid and/or application of acidic primers or adhesive resins [5-9]. MMPs, along with cysteine cathepsins that are capable of activating MMPs and of cleaving type I collagen, have been shown to be responsible for the hydrolytic degradation of the collagen matrix within the hybrid layer $[5,10]$. Adhesive retention to dentin relies on infiltration of resin into the mineralized dentin. This infiltration requires removal of minerals by phosphoric acid or acidic monomers. The minerals are replaced by the water used to rinse off the phosphoric acid (etch-and-rinse adhesive systems) or by the water used as a solvent in the primers and/or adhesive resins. During application of the adhesive resin, solvated monomers are intended to replace the water and penetrate into and around collagen fibrils to result in hybridization [11]. Unfortunately, adhesive resins are not able to replace all water, and the bottom portion of the hybrid layer contains collagen fibrils that are only partially protected by resin $[12,13]$. The water remnants and incomplete resin impregnation render the collagen fibrils and the hydrophilic resins vulnerable to hydrolytic degradation, the destruction of collagen fibrils being caused mainly by activation of the collagen-bound MMPs $[3,9,11]$ and that of the resins by 
degradation of the ester bonds in the adhesive polymer. The gradual destruction of the hybrid layer is inevitably accompanied by a gradual loss of bond strength.

In an effort to retard endogenous enzymatic degradation of the resin-dentin bonds, numerous studies have explored the capacity of various protease inhibitors such as chlorhexidine and quaternary ammonium compounds for their ability to inhibit MMP activity. These inhibitors, either incorporated in the phosphoric acid used prior to application of etch-andrinse adhesive systems or applied as a separate step after phosphoric acid etching, have been shown to inhibit MMPs and cysteine cathepsins, to prevent collagen degradation and preserve the integrity of hybrid layer collagen matrix [6, 14-19], and to reduce the time-dependent deterioration of the resin-dentin bond [17, 20-25]. Chlorhexidine and the quaternary ammonium compound benzalkonium chloride (BAC) have also been incorporated into adhesive primers $[16,26]$ or into the adhesive component itself $[27,28]$, thus avoiding an extra step in the application procedure and possibly prolonging their presence in the hybrid layer. When incorporated into primers/adhesives, both protease inhibitors were still capable of reducing collagen degradation within the hybrid layer and retarding bond deterioration, especially in relatively hydrophilic primers/adhesives [27] and provided that the concentration was sufficiently high [26]. Incorporation of $1 \% \mathrm{BAC}$ even led to a higher immediate bond strength than was obtained with the control adhesive [28].

Bond strength to eroded dentin has been found not only to be lower than the bond strength to normal, sound dentin but also to be more adversely affected by aging [29]. Eroded teeth displaying exposed dentin very often need treatment with sealants or resin composite in order to prevent further loss of tooth substance [30-33]. Any improvement in bond strength to eroded dentin and in bond durability would therefore be of great clinical interest. Consequently, this study aimed to investigate the effect of incorporation of BAC on long-term bond strength of a two-step etch-and-rinse and a three-step etch-and-rinse adhesive system to normal or artificially eroded dentin.

The null hypotheses were that neither (1) BAC incorporation nor (2) type of dentin (i.e., normal or eroded) or (3) duration of storage (i.e., 24 hours or one year) would have any influence on bond strength for either adhesive system.

\section{Materials and Methods}

2.1. Preparation of Dentin Specimens. A total of 256 dentin specimens were prepared from 128 extracted human permanent molars without restorations or caries $(n=16$ dentin specimens/group, 16 groups: two adhesive systems without/with BAC incorporation on normal or artificially eroded dentin stored for either $24 \mathrm{~h}$ or 1 year). Before extraction, patients had been informed about the use of the molars for research purposes and verbal consent had been obtained. After extraction, the molars were pooled. The local ethics committee categorizes pooled teeth as an "irreversibly anonymized biobank" and thus no previous ethical approval was needed. The molars were cleaned under tap water with a
TABLE 1: Composition of the de- and remineralization solutions used in the artificial erosion protocol.

\begin{tabular}{cc}
\hline Solution (at $\left.37^{\circ} \mathrm{C}\right)$ & Composition \\
\hline Demineralization & $\begin{array}{c}\text { 1\% citric acid with pH of 3.5 (anhydrous citric } \\
\text { acid; Merck, Darmstadt, Germany) }\end{array}$ \\
\hline & $0.002 \mathrm{~g}$ ascorbic acid, $0.58 \mathrm{~g} \mathrm{NaCl}, 0.17 \mathrm{~g}$ \\
& $\mathrm{CaCl}_{2}, 0.16 \mathrm{~g} \mathrm{NH}_{4} \mathrm{Cl}, 1.27 \mathrm{~g} \mathrm{KCl}, 0.16 \mathrm{~g}$ \\
Remineralization & $\mathrm{NaSCN}_{0} 0.33 \mathrm{~g} \mathrm{KH}_{2} \mathrm{PO}_{4}, 0.34 \mathrm{~g} \mathrm{Na}_{2} \mathrm{HPO}_{4}$ \\
& dissolved in $1 \mathrm{~L}$ of demineralized water; $\mathrm{pH}$ is \\
& set to 6.4 with $\mathrm{HCl}$ \\
\hline
\end{tabular}

scaler to remove any debris and soft tissue and stored in $2 \%$ chloramine solution in the refrigerator $\left(4^{\circ} \mathrm{C}\right)$ until needed.

For preparation of dentin specimens, the molars were apically shortened with a water-cooled diamond saw (IsoMet Low Speed Saw, Buehler; Lake Bluff, IL, USA) and then sectioned along the mesiodistal axis (IsoMet Low Speed Saw, Buehler) and finally wet-ground from the buccal and oral surfaces to obtain flat dentin surfaces. Grinding was performed with grit \#220 followed by grit \#500 silicon carbide (SiC) abrasive papers on a Struers LaboPol-21 grinding machine (Struers; Ballerup, Denmark). The oral and buccal halves of the molars were embedded in cylindrical stainless steel molds with self-curing acrylic resin (Paladur, Heraeus Kulzer GmbH, Hanau, Germany). Consequently, two dentin specimens per molar were obtained, and after removal of the steel molds, all dentin specimens were kept in a humid chamber $\left(100 \%\right.$ humidity) in the refrigerator $\left(4^{\circ} \mathrm{C}\right)$. Before adhesive treatment and preparation of shear bond strength (SBS) specimens, one dentin specimen (i.e., one half) of each molar underwent artificial erosion.

2.2. Artificial Erosion of Dentin Specimens. The 128 dentin specimens that underwent artificial erosion were subjected to a cyclic de- and remineralization procedure over 7 days with 42 de- and remineralization cycles in analogy to a previous study [29] ( 6 cycles per $24 \mathrm{~h}, 4 \mathrm{~h}$ per cycle with $5 \mathrm{~min}$ demineralization, $3.5 \mathrm{~h}$ remineralization (remaining $25 \mathrm{~min}$ : rinsing with deionized water between each demineralization/remineralization)). The cyclic de- and remineralization procedure was performed in a custom-made $\mathrm{pH}$-cycling machine and the composition of the de- and remineralization solutions [34] is listed in Table 1 . The $\mathrm{pH}$ of the de- and remineralization solutions was checked daily. After the cyclic de- and remineralization procedure, all artificially eroded dentin specimens were kept in a humid chamber $(100 \%$ humidity) in the refrigerator $\left(4^{\circ} \mathrm{C}\right)$.

2.3. Preparation of SBS Specimens. One hour before adhesive treatment, the dentin specimens were retrieved from the refrigerator and kept in tap water at room temperature. The dentin surfaces of the artificially eroded halves of each molar were left untreated whereas the dentin surfaces of the other halves were wet-ground for $5 \mathrm{~s}$ with grit \#500 $\mathrm{SiC}$ abrasive papers (Struers) to obtain a standardized smear layer, with the abrasive papers being changed after grinding of 8 dentin specimens. Subsequently, each dentin specimen was air-dried 
TABLE 2: Adhesive systems and adhesive treatment (BAC: benzalkonium chloride).

\begin{tabular}{|c|c|c|}
\hline \multirow{6}{*}{$\begin{array}{l}\text { Adper Scotchbond } 1 X T \\
(3 \mathrm{M} \text { ESPE, St. Paul, MN, USA) }\end{array}$} & $\begin{array}{l}\text { (1) Phosphoric acid etching } \\
\text { (Scotchbond Universal Etchant; lot number: } 568712 ; 35 \% \text { phosphoric acid) }\end{array}$ & $15 \mathrm{~s}$ \\
\hline & Water spray & $10 \mathrm{~s}$ \\
\hline & Blot dry (cotton pellet) & - \\
\hline & $\begin{array}{l}\text { (2) Adper Scotchbond 1XT ( } 3 \text { coats; lot number: N597610; BisGMA, HEMA, } \\
\text { dimethacrylates, a methacrylate functional copolymer of polyacrylic and } \\
\text { polyitaconic acids, ethanol, water, initiators, silane treated silica) } \\
\text { (i) without/with } 1 \% \text { BAC }\end{array}$ & $15 \mathrm{~s}$ \\
\hline & Short air dry & $(\sim 5 s)$ \\
\hline & (3) Light-cure & $10 \mathrm{~s}$ \\
\hline \multirow{8}{*}{$\begin{array}{l}\text { OptiBond FL } \\
\text { (KerrHawe, Scafati, Italy) }\end{array}$} & $\begin{array}{l}\text { (1) Phosphoric acid etching } \\
\text { (Kerr Gel Etchant; lot number: 5329366; 37.5\% phosphoric acid) }\end{array}$ & $15 \mathrm{~s}$ \\
\hline & Water spray & $>15 \mathrm{~s}$ \\
\hline & Air dry & $>3 \mathrm{~s}$ \\
\hline & $\begin{array}{l}\text { (2) OptiBond Prime (lot number: 5296441; HEMA, GPDM, MMEP, ethanol, water, } \\
\text { photoinitiator) } \\
\text { (i) without/with } 1 \% \text { BAC }\end{array}$ & $15 \mathrm{~s}$ \\
\hline & Short air dry & $(\sim 5 \mathrm{~s})$ \\
\hline & $\begin{array}{l}\text { (3) OptiBond Adhesive (lot number: 5344022; BisGMA, HEMA, GPDM, } \\
\text { photoinitiator, filler) }\end{array}$ & $15 \mathrm{~s}$ \\
\hline & Short air dry & $(\sim 3 s)$ \\
\hline & (4) Light-cure & $10 \mathrm{~s}$ \\
\hline
\end{tabular}

and the bonding area defined and isolated by use of selfadhesive tape with a perforation (diameter $\sim 2 \mathrm{~mm}$ ). The bonding area was then treated as listed in Table 2. For Adper Scotchbond 1XT and OptiBond FL with BAC, $60 \mathrm{mg}$ benzalkonium chloride BioXtra (Sigma-Aldrich, St. Louis, MO, USA; CAS number: 63449-41-2, lot number: BCBP1900V) was incorporated into $6 \mathrm{~g}$ Adper Scotchbond 1XT and into $6 \mathrm{~g}$ OptiBond FL Prime with the use of a precision balance (Sartorius R180D, Sartorius, Göttingen, Germany) leading to a BAC concentration of $1 \%$ [28]. After the adhesive treatment, a split Teflon mold (inner diameter $1.5 \mathrm{~mm} \approx$ bonding area $1.8 \mathrm{~mm}^{2}$; height: $2 \mathrm{~mm}$ ) was clamped to the dentin surface and filled with resin composite (Filtek Z250, 3M ESPE; St. Paul, MN, USA; shade A3, lot number: N605143). The resin composite was covered with a Mylar strip and light-cured for $20 \mathrm{~s}$. All light-curing was performed with an LED-curing unit (Demi, Kerr Corporation, Middleton, WI, USA), and at the beginning and end of each day of specimen preparation the light power density was verified with a radiometer (Demetron L.E.D. Radiometers, Kerr Corporation) to be at least $1000 \mathrm{~mW} / \mathrm{cm}^{2}$. After light-curing, the SBS specimens were placed in a black photoresistant box in order to avoid any further influence of ambient light. Five minutes after completion of light-curing, the specimens were freed from the Teflon mold. All SBS specimens were then stored in black photoresistant boxes in an incubator (Memmert UM 500, Memmert \& Co., Schwabach, Germany) at $37^{\circ} \mathrm{C}$ and $100 \%$ humidity for $24 \mathrm{~h}$. SBS specimens prepared for 1-year storage were transferred to tap water and kept in the incubator at $37^{\circ} \mathrm{C}$, with the tap water being changed periodically.

2.4. SBS Testing and Failure Mode Determination. After storage, all specimens were subjected to SBS testing by use of a wire (stainless steel, diameter $0.6 \mathrm{~mm}$ ) at a cross-head speed of $1 \mathrm{~mm} / \mathrm{min}$ in a universal testing machine (Zwick Z1.0 TN, Zwick GmbH \& Co. KG, Ulm, Germany). The maximum force $\left(F_{\max }[\mathrm{N}]\right)$ was recorded and the SBS values $(\mathrm{MPa})$ were calculated $\left(F_{\max }[\mathrm{N}] /\right.$ bonding area $\left.\left[\mathrm{mm}^{2}\right]\right)$ resulting in $16 \mathrm{SBS}$ values per group for statistical analysis.

After SBS testing, the failure mode of each specimen was determined under a stereomicroscope (Leica ZOOM 2000, Leica, Buffalo, NY, USA) at 40x magnification and classified as (1) cohesive failure in dentin, (2) adhesive failure at dentin/adhesive interface, (3) adhesive failure at adhesive/resin composite interface, (4) cohesive failure in resin composite, or (5) mixed failure (combinations of failure modes 1 to 4 ).

2.5. Statistical Analysis. Due to a lack of normal distribution (Shapiro Wilk's test, $p=0.0012$ ), SBS values were analyzed with a nonparametric ANOVA followed by Bonferroni Holm correction for multiple testing. For post hoc analysis, Wilcoxon-Mann-Whitney tests were applied without Bonferroni Holm correction. All calculations were performed with $R$ version 3.3.0 (the $R$ Foundation for Statistical Computing, Vienna, Austria, https://www.R-project.org) after the significance level had been set at $\alpha=0.05$. Failure modes after SBS testing were analyzed descriptively.

\section{Results}

The SBS values of the two adhesive systems (each without/with 1\% BAC incorporation) and the four experimental conditions (i.e., normal/artificially eroded dentin and $24 \mathrm{~h} / 1$ year storage) are depicted in Figure 1 for Adper Scotchbond 1XT and in Figure 2 for OptiBond FL. The nonparametric 


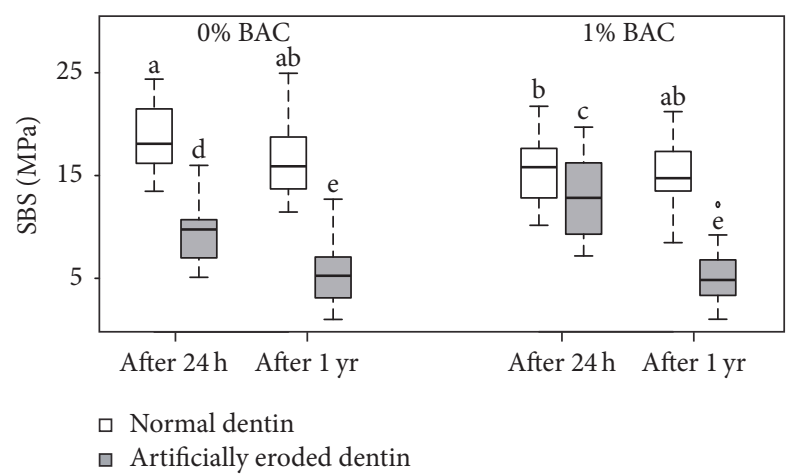

FIGURE 1: Shear bond strength (SBS (MPa); medians, lower and upper quartiles, and minima and maxima) of Adper Scotchbond 1XT (without/with 1\% benzalkonium chloride (BAC) and the four experimental conditions (i.e., normal/artificially eroded dentin and 24 h/1-year (yr) storage); $n=16$ /group). Different lowercase letters show significant differences between the groups.

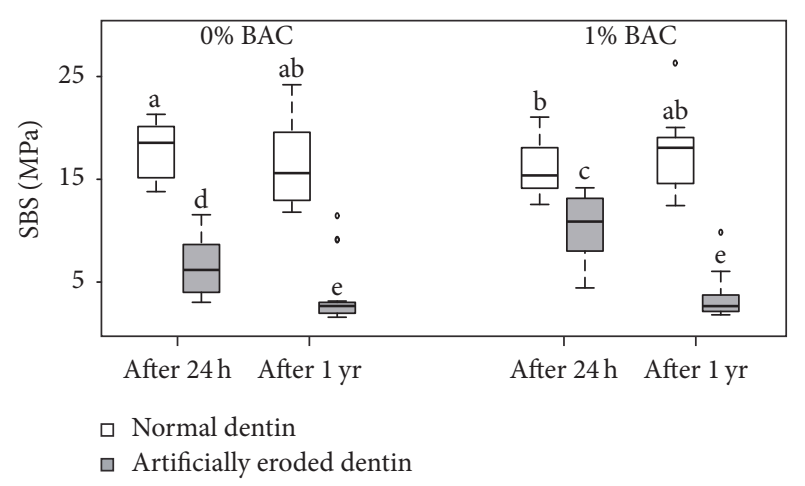

FIGURE 2: Shear bond strength (SBS (MPa); medians, lower and upper quartiles, and minima and maxima) of OptiBond FL (without/with $1 \%$ benzalkonium chloride (BAC) and the four experimental conditions (i.e., normal/artificially eroded dentin and $24 \mathrm{~h} / 1$ year (yr) storage); $n=16$ /group). Different lowercase letters show significant differences between the groups.

ANOVA showed a significant effect of the factors "type of dentin" (i.e., normal or artificially eroded) and "duration of storage" (i.e., $24 \mathrm{~h}$ or 1 year) (both $p<0.001$ ) but no significant effect of the factors "BAC incorporation" ( $p=$ $1.000)$ and "adhesive system" ( $p=0.397)$. The ANOVA also showed significant twofold interactions between the factors "type of dentin"/"BAC incorporation" ( $p=0.002)$, "type of dentin"/"duration of storage" $(p=0.0003)$, and "type of dentin"/"adhesive system" ( $p=0.009)$ as well as a significant threefold interaction between the factors "type of dentin"/“BAC incorporation"/“duration of storage" $(p<0.001)$. All remaining interactions were not significant $(p \geq 0.927)$. The significant interaction in "type of dentin"/"adhesive system" ( $p=0.009)$ implied that the two adhesive systems yielded statistically similar SBS to normal dentin (median SBS values (MPa) without BAC at 24 h/1 year: Adper Scotchbond 1XT 18.1/15.9; OptiBond FL 18.6/15.6) and Adper Scotchbond 1XT yielded higher SBS to eroded dentin than did OptiBond FL (median SBS values
(MPa) without BAC at $24 \mathrm{~h} / 1$ year: Adper Scotchbond $1 \mathrm{XT}$ 9.8/5.3; OptiBond FL 6.2/2.7). However, because of the not statistically significant main effect of adhesive system, the SBS values obtained with Adper Scotchbond 1XT and OptiBond FL were pooled for further post hoc tests. These tests revealed that SBS was significantly higher for normal dentin than for artificially eroded dentin regardless of BAC incorporation and/or duration of storage $(p<0.001)$. On normal dentin, BAC incorporation led to significantly lower SBS after $24 \mathrm{~h}$ $(p=0.018)$, but to statistically similar SBS after 1 year $(p$ $=1.000)$. On artificially eroded dentin, BAC incorporation led to significantly higher SBS after $24 \mathrm{~h}(p=0.001)$ but to statistically similar SBS after 1 year $(p=1.000)$. Storage for 1 year resulted in a reduction in SBS to artificially eroded dentin $(p \leq 0.001)$, whereas SBS to normal dentin remained stable $(p \geq 0.082)$.

Of the five afore-listed failure modes, only cohesive failures in dentin (Figure 3(a)), adhesive failures at dentin/ adhesive interface (Figure 3(b)), and mixed failures (combinations of cohesive failures in dentin and adhesive failures at dentin/adhesive interface, Figure 3(c)) occurred. The distribution of failure modes after SBS testing is shown in Table 3. For both adhesive systems and both storage durations, the predominant failure mode was adhesive failure at the dentin/adhesive interface with a tendency to even more adhesive failures after 1-year storage.

\section{Discussion}

The present study investigated the effect of benzalkonium chloride (BAC) incorporation into a two-step (Adper Scotchbond 1XT) and a three-step (OptiBond FL) etch-and-rinse adhesive system on the long-term bond strength to normal and to artificially eroded dentin. Since BAC incorporation under certain experimental conditions led to significantly different bond strengths than did the non-BAC-containing control groups, the first null hypothesis was rejected. On normal dentin, BAC incorporation led to a reduction in the $24 \mathrm{~h}$ bond strength and a slight increase in the number of adhesive failures at the dentin/adhesive interface, indicating that the incorporation of BAC per se affected the bondpromoting capacity of the two adhesive systems. It may be that the incorporation of BAC upset the optimized monomersolvent balance in the adhesive, thereby either hampering effective infiltration into and around the collagen fibrils [16] or interfering with the light-induced polymerization to decrease the degree of cure [35]. The result is in contrast to that of Sabatini et al. who even reported a favorable effect on the $24 \mathrm{~h}$ microtensile bond strength of adding $1 \%$ BAC to the Adper Single Bond Plus adhesive [28]. One explanation for the discrepancy between the two studies is a change in the composition from that of Adper Single Bond Plus to that of Adper Scotchbond 1XT; another is the difference in dentin tubule orientation of the dentin specimens used. Whereas Sabatini et al. [28] performed bonding to occlusal dentin (dentin tubules cut perpendicularly), the present study performed bonding to axial dentin (dentin tubules cut parallel). It has been speculated that occlusal orientation of dentin tubules facilitates migration of the water present in 


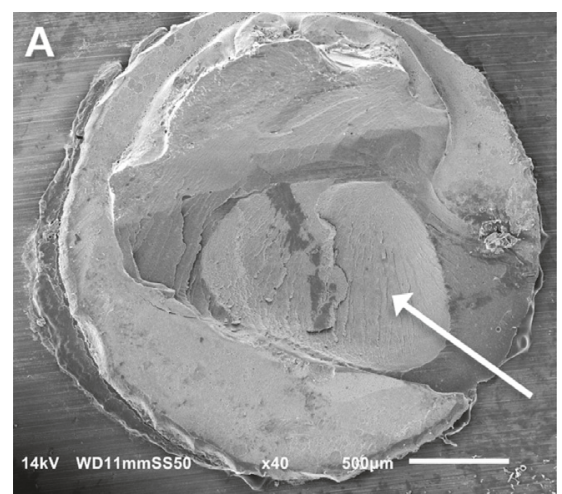

(a)

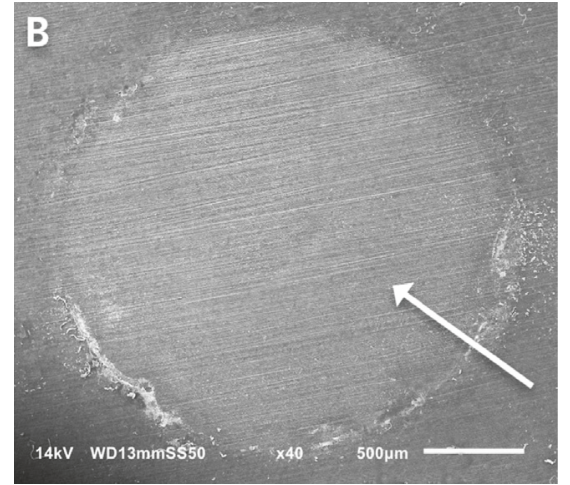

(b)

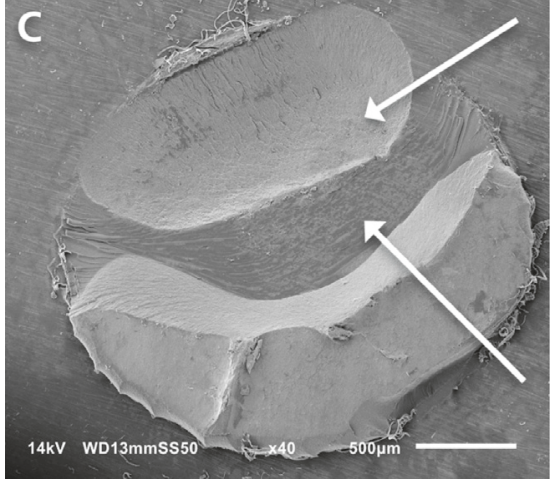

(c)

FIGURE 3: Representative scanning electron micrographs of the three failure modes observed: (a) cohesive failure in dentin, (b) adhesive failure at dentin/adhesive interface, and (c) mixed failure (combination of cohesive failure in dentin and adhesive failure at dentin/adhesive interface with remnants of adhesive system/resin composite).

the tubules to the adhesive interface [36]. Sabatini et al. [28] argued that BAC incorporation into the adhesive improves diffusion and infiltration of the adhesive into the waterfilled spaces between the collagen fibrils and dentin tubules. Increased exposure to water from the dentin tubules might then explain why incorporation of BAC into the adhesive had a positive effect on occlusal dentin as opposed to axial and less "wet" dentin.

The artificial erosion protocol applied in the present study led to significantly lower bond strengths to dentin and to a tendency to more adhesive failures at the dentin/adhesive interface, regardless of BAC incorporation and/or duration of storage and thus to rejection of the second null hypothesis. This result corroborates the bond strength findings of Zimmerli et al. [29] who, using TEM and the exact same artificial erosion protocol as in the present study, also reported a thicker layer of exposed collagen that could hardly be infiltrated by the adhesives applied. Probable reasons presented for the inefficient hybridization were collapse of the demineralized collagen fibrils and an increased water content that not only prevented the adhesive from fully infiltrating the demineralized zone but also hampered proper polymerization of the adhesive. Thus, it is likely that the lower $24 \mathrm{~h}$ bond strength to eroded dentin found in the present study can be attributed to inferior hybridization. The fact that Adper Scotchbond 1XT yielded higher bond strength to eroded dentin than did OptiBond FL seems to reflect a superior capacity of the Adper Scotchbond 1XT adhesive to infiltrate thick demineralization zones and to effectively polymerize in humid conditions. The relatively good performance on eroded dentin is corroborated by studies on its predecessor Adper Single Bond $2[37,38]$.

Whereas BAC incorporation, as mentioned above, caused a reduction in the $24 \mathrm{~h}$ bond strength to normal dentin, a positive effect of BAC incorporation on the $24 \mathrm{~h}$ bond strength to eroded dentin was found. As previously mentioned, Sabatini et al. [28] speculated that BAC incorporation into the adhesive improves diffusion and infiltration of the adhesive into the water-filled spaces between the collagen fibrils and dentin tubules, an improvement that could be argued to have greater effect on eroded dentin displaying a thicker demineralization zone.

Both etch-and-rinse adhesive systems provided bonds to normal dentin that remained stable over the study period whether or not BAC had been incorporated into the adhesive. As regards the three-step etch-and-rinse adhesive system OptiBond FL, this finding is in agreement with previous studies that, according to the review by de Munck et al., have reported its bonding effectiveness to be unaffected by water storage, thermocycling, and/or mechanical loading [2]. The resistance to bond degradation of OptiBond FL, and other three-step etch-and-rinse adhesive systems, has been attributed to the inclusion of a separate and hydrophobic adhesive resin layer that is presumed to prevent, or at least decrease, the absorption of water within the dentin matrix and thus limit the degradation of collagen by MMPs [39]. Since MMPs are enzymes that require water to hydrolyze the peptide bonds in the collagen molecules [6], it may be that, in the presence of a hydrophobic adhesive resin layer, sealing the acid-etched dentin not only renders the bonded interface more stable but also lets a lower amount of water diffuse through the hybrid layer whereby the collagen hydrolysis by MMPs would be limited [40] and the MMP-inhibiting effect of BAC would not be expressed or would be rendered obsolete. The bond of two-step etchand-rinse adhesive systems such as Adper Scotchbond 1XT and which do not comprise a separate adhesive layer has generally proved to be less durable, and the positive result found with this adhesive system is thus in conflict with two previous studies $[4,28]$. Possible explanations for the fact that Adper Scotchbond 1XT resisted bond degradation are (1) an upgraded formulation as compared to previous versions (e.g., the Adper Single Bond Plus employed by Sabatini et al. [28] that, based on continuing improved understanding on dentin bonding, displayed more complete hybridization) and (2) the test method. Since most degradation processes are diffusion-rate-dependent, the length of the diffusion path is as important a parameter as the diffusion time. Considering 
TABLE 3: Distribution of failure modes after shear bond strength testing ( $n=16 /$ group; BAC: benzalkonium chloride).

\begin{tabular}{|c|c|c|c|}
\hline $\begin{array}{l}\text { Adhesive system } \\
\text { Dentin }\end{array}$ & $\begin{array}{l}\text { Cohesive failures in dentin } \\
(\%)\end{array}$ & $\begin{array}{l}\text { Adhesive failures at dentin/adhesive } \\
\text { interface (\%) }\end{array}$ & Mixed failures (\%) \\
\hline \multicolumn{4}{|c|}{ After 24-hour storage } \\
\hline \multicolumn{4}{|c|}{ Adper Scotchbond $1 \mathrm{XT}$ without $1 \%$ BAC } \\
\hline Normal dentin & 12.5 & 62.5 & 25 \\
\hline \multicolumn{4}{|c|}{ Adper Scotchbond $1 \mathrm{XT}$ without $1 \%$ BAC } \\
\hline Artificially eroded dentin & 0 & 100 & 0 \\
\hline \multicolumn{4}{|c|}{ Adper Scotchbond $1 \mathrm{XT}$ with $1 \%$ BAC } \\
\hline Normal dentin & 0 & 87.5 & 12.5 \\
\hline \multicolumn{4}{|c|}{ Adper Scotchbond 1XT with $1 \%$ BAC } \\
\hline Artificially eroded dentin & 0 & 87.5 & 12.5 \\
\hline \multicolumn{4}{|l|}{ OptiBond FL without $1 \%$ BAC } \\
\hline Normal dentin & 6.25 & 75 & 18.75 \\
\hline \multicolumn{4}{|l|}{ OptiBond FL without $1 \%$ BAC } \\
\hline Artificially eroded dentin & 0 & 100 & 0 \\
\hline \multicolumn{4}{|l|}{ OptiBond FL with $1 \%$ BAC } \\
\hline Normal dentin & 6.25 & 87.5 & 6.25 \\
\hline \multicolumn{4}{|l|}{ OptiBond FL with $1 \%$ BAC } \\
\hline Artificially eroded dentin & 0 & 100 & 0 \\
\hline \multicolumn{4}{|c|}{ After 1-year storage } \\
\hline \multicolumn{4}{|c|}{ Adper Scotchbond $1 \mathrm{XT}$ without $1 \%$ BAC } \\
\hline Normal dentin & 6.25 & 75 & 18.75 \\
\hline \multicolumn{4}{|c|}{ Adper Scotchbond $1 \mathrm{XT}$ without $1 \%$ BAC } \\
\hline Artificially eroded dentin & 0 & 100 & 0 \\
\hline \multicolumn{4}{|c|}{ Adper Scotchbond 1XT with 1\% BAC } \\
\hline Normal dentin & 6.25 & 93.75 & 0 \\
\hline \multicolumn{4}{|c|}{ Adper Scotchbond 1XT with 1\% BAC } \\
\hline Artificially eroded dentin & 0 & 100 & 0 \\
\hline \multicolumn{4}{|l|}{ OptiBond FL without 1\% BAC } \\
\hline Normal dentin & 0 & 81.25 & 18.75 \\
\hline \multicolumn{4}{|l|}{ OptiBond FL without $1 \%$ BAC } \\
\hline Artificially eroded dentin & 0 & 100 & 0 \\
\hline \multicolumn{4}{|l|}{ OptiBond FL with $1 \%$ BAC } \\
\hline Normal dentin & 0 & 93.75 & 6.25 \\
\hline \multicolumn{4}{|l|}{ OptiBond FL with $1 \%$ BAC } \\
\hline Artificially eroded dentin & 0 & 100 & 0 \\
\hline
\end{tabular}

that the diameter of the bonded area was $1.5 \mathrm{~mm}$, which is more than the $1 \mathrm{~mm}$ width or breadth of most sticks or beams applied in the much used microtensile strength test, it could be argued that water may not have had access to the entire bonding surface, excluding bond degradation at the center of the bond strength specimen. However, the storage time in the present study was much longer than that in several studies, which found a decline in bond strength.

In contrast to the bond strength to normal dentin, the bond strength to eroded dentin deteriorated after 1-year water storage, and the third null hypothesis was therefore partially rejected. The fact that the bond to eroded dentin decreased in consequence of the 1-year water storage corroborates the findings of Zimmerli et al. who reported that bond strength to eroded dentin was more adversely affected by aging [29]. The inferior hybridization mentioned above explains not only the lower immediate bond strength obtained to eroded dentin but also the reduced bond stability caused by enhanced hydrolytic degradation of the imperfect hybrid layer. The lack of ability of BAC incorporation to improve bond stability to eroded dentin may have the following explanation: nonpolymerizable MMP inhibitors, such as BAC and chlorhexidine, bind to dentin electrostatically [41], and noncovalently bound molecules may leach out of the hybrid layer. Leaching of the incorporated BAC would have weakened its MMPinhibitory effect and minimized or annihilated any positive 
effect on bond durability. The fact that Sabatini et al. [28] found incorporation of BAC to ensure bond stability may be explained by the 6 months' storage, that is, only half of the 1year storage in the present study, and in less leaching of the unbound BAC.

The positive result of BAC incorporation on the stability of the bond to normal dentin reported by Sabatini et al. [28] was not confirmed on artificially eroded dentin in the present study. In an effort to gain an increased understanding of the underlying mechanisms for bond durability, future studies should analyze the bonding interfaces on the two dentin substrates and compare adhesives modified with BAC with control adhesives. Considering the discouraging result on eroded dentin, it may be more promising to pursue long-term stability of resin-dentin bonds by (polymerizable) collagen crosslinks $[42,43]$.

\section{Conclusions}

Based on the results of the present in vitro study, the following conclusions can be drawn:

(i) Bond strength was significantly lower to artificially eroded dentin than to normal dentin.

(ii) Bond strength to normal dentin did not deteriorate during the 1-year storage.

(iii) Bond strength to artificially eroded dentin deteriorated during the 1-year storage.

(iv) Incorporation of BAC did not increase bond durability.

Consequently, benzalkonium chloride incorporated into the adhesive of a two-step and a three-step adhesive system did not improve bond stability. Other measures to prevent bond degradation and to ensure long-term survival of resin-dentin bonds must be explored.

\section{Ethical Approval}

Before extraction of the permanent human molars, patients had been informed about the use of the molars for research purposes and verbal consent had been obtained. After extraction, the molars were pooled. The local ethics committee categorizes pooled teeth as an "irreversibly anonymized biobank" and thus no previous ethical approval was needed.

\section{Conflicts of Interest}

The authors declare that they have no conflicts of interest, real or perceived, financial or nonfinancial.

\section{Acknowledgments}

The present study was funded by the authors' own institution. The authors would like to thank the companies for providing the materials needed. Furthermore, the authors also thank G. Fischer, Institute of Mathematical Statistics and Actuarial Science, University of Bern, for the statistical analysis.

\section{References}

[1] Y. Shono, M. Terashita, J. Shimada et al., "Durability of resindentin bonds," Journal of Adhesive Dentistry, vol. 1, no. 3, pp. 211-218, 1999.

[2] J. de Munck, K. van Landuyt, M. Peumans et al., "A critical review of the durability of adhesion to tooth tissue: methods and results," Journal of Dental Research, vol. 84, no. 2, pp. 118$132,2005$.

[3] L. Breschi, A. Mazzoni, A. Ruggeri, M. Cadenaro, R. Di Lenarda, and E. de Stefano Dorigo, "Dental adhesion review: aging and stability of the bonded interface," Dental Materials, vol. 24, no. 1, pp. 90-101, 2008.

[4] J. De Munck, A. Mine, A. Poitevin et al., "Meta-analytical review of parameters involved in dentin bonding," Journal of Dental Research, vol. 91, no. 4, pp. 351-357, 2012.

[5] L. Tjäderhane, F. D. Nascimento, L. Breschi et al., "Optimizing dentin bond durability: control of collagen degradation by matrix metalloproteinases and cysteine cathepsins," Dental Materials, vol. 29, no. 1, pp. 116-135, 2013.

[6] D. H. Pashley, F. R. Tay, C. Yiu et al., "Collagen degradation by host-derived enzymes during aging," Journal of Dental Research, vol. 83, no. 3, pp. 216-221, 2004.

[7] Y. Nishitani, M. Yoshiyama, B. Wadgaonkar et al., "Activation of gelatinolytic/collagenolytic activity in dentin by self-etching adhesives," European Journal of Oral Sciences, vol. 114, no. 2, pp. 160-166, 2006.

[8] F. R. Tay, D. H. Pashley, R. J. Loushine, R. N. Weller, F. Monticelli, and R. Osorio, "Self-etching adhesives increase collagenolytic activity in radicular dentin," Journal of Endodontics, vol. 32, no. 9, pp. 862-868, 2006.

[9] A. Mazzoni, F. D. Nascimento, M. Carrilho et al., "MMP activity in the hybrid layer detected with in situ zymography," Journal of Dental Research, vol. 91, no. 5, pp. 467-472, 2012.

[10] M. Hashimoto, "A review-micromorphological evidence of degradation in resin-dentin bonds and potential preventional solutions," Journal of Biomedical Materials Research Part B: Applied Biomaterials, vol. 92, no. 1, pp. 268-280, 2010.

[11] D. H. Pashley, F. R. Tay, L. Breschi et al., "State of the art etchand-rinse adhesives," Dental Materials, vol. 27, no. 1, pp. 1-16, 2011.

[12] S. R. Armstrong, J. C. Keller, and D. B. Boyer, "Mode of failure in the dentin-adhesive resin-resin composite bonded joint as determined by strength-based ( $\mu \mathrm{TBS}$ ) and fracturebased (CNSB) mechanical testing," Dental Materials, vol. 17, no. 3, pp. 201-210, 2001.

[13] L. Breschi, C. Prati, P. Gobbi et al., "Immunohistochemical analysis of collagen fibrils within the hybrid layer: a FEISEM study," Operative Dentistry, vol. 29, no. 5, pp. 538-546, 2004.

[14] J. Hebling, D. H. Pashley, L. Tjäderhane, and F. R. Tay, "Chlorhexidine arrests subclinical degradation of dentin hybrid layers in vivo," Journal of Dental Research, vol. 84, no. 8, pp. 741746, 2005.

[15] W. W. Brackett, F. R. Tay, M. G. Brackett, A. Dib, R. J. Sword, and D. H. Pashley, "The effect of chlorhexidine on dentin hybrid layers in vivo," Operative Dentistry, vol. 32, no. 2, pp. 107-111, 2007.

[16] J. De Munck, P. E. Van Den Steen, A. Mine et al., "Inhibition of enzymatic degradation of adhesive-dentin interfaces," Journal of Dental Research, vol. 88, no. 12, pp. 1101-1106, 2009. 
[17] L. Breschi, A. Mazzoni, F. Nato et al., "Chlorhexidine stabilizes the adhesive interface: a 2 -year in vitro study," Dental Materials, vol. 26, no. 4, pp. 320-325, 2010.

[18] A. Tezvergil-Mutluay, M. M. Mutluay, L.-S. Gu et al., "The antiMMP activity of benzalkonium chloride," Journal of Dentistry, vol. 39, no. 1, pp. 57-64, 2011.

[19] A. Tezvergil-Mutluay, K. A. Agee, T. Uchiyama et al., "The inhibitory effects of quaternary ammonium methacrylates on soluble and matrix-bound MMPs," Journal of Dental Research, vol. 90, no. 4, pp. 535-540, 2011.

[20] M. R. O. Carrilho, S. Geraldeli, F. Tay et al., "In vivo preservation of the hybrid layer by chlorhexidine," Journal of Dental Research, vol. 86, no. 6, pp. 529-533, 2007.

[21] L. Breschi, F. Cammelli, E. Visintini et al., "Influence of chlorhexidine concentration on the durability of etch-and-rinse dentin bonds: a 12-month in vitro study," Journal of Adhesive Dentistry, vol. 11, no. 3, pp. 191-198, 2009.

[22] E. A. Campos, G. M. Correr, D. P. Leonardi, F. Barato-Filho, C. C. Gonzaga, and J. C. Zielak, "Chlorhexidine diminishes the loss of bond strength over time under simulated pulpal pressure and thermo-mechanical stressing," Journal of Dentistry, vol. 37, no. 2, pp. 108-114, 2009.

[23] A. D. Loguercio, R. Stanislawczuk, L. G. Polli, J. A. Costa, M. D. Michel, and A. Reis, "Influence of chlorhexidine digluconate concentration and application time on resin-dentin bond strength durability," European Journal of Oral Sciences, vol. 117, no. 5, pp. 587-596, 2009.

[24] R. Stanislawczuk, A. Reis, and A. D. Loguercio, "A 2-year in vitro evaluation of a chlorhexidine-containing acid on the durability of resin-dentin interfaces," Journal of Dentistry, vol. 39, no. 1, pp. 40-47, 2011.

[25] R. Stanislawczuk, R. C. Amaral, C. Zander-Grande, D. Gagler, A. Reis, and A. D. Loguercio, "Chlorhexidine-containing acid conditioner preserves the longevity of resin-dentin bonds," Operative Dentistry, vol. 34, no. 4, pp. 481-490, 2009.

[26] J. Zhou, J. Tan, L. Chen, D. Li, and Y. Tan, “The incorporation of chlorhexidine in a two-step self-etching adhesive preserves dentin bond in vitro," Journal of Dentistry, vol. 37, no. 10, pp. 807-812, 2009.

[27] C. K. Y. Yiu, N. Hiraishi, F. R. Tay, and N. M. King, "Effect of chlorhexidine incorporation into dental adhesive resin on durability of resin-dentin bond," Journal of Adhesive Dentistry, vol. 14, no. 4, pp. 355-362, 2012.

[28] C. Sabatini, J. H. Kim, and P. Ortiz Alias, "In vitro evaluation of benzalkonium chloride in the preservation of adhesive interfaces," Operative Dentistry, vol. 39, no. 3, pp. 283-290, 2014.

[29] B. Zimmerli, J. De Munck, A. Lussi, P. Lambrechts, and B. Van Meerbeek, "Long-term bonding to eroded dentin requires superficial bur preparation," Clinical Oral Investigations, vol. 16, no. 5, pp. 1451-1461, 2012.

[30] A. Azzopardi, D. W. Bartlett, T. F. Watson, and M. Sherriff, “The measurement and prevention of erosion and abrasion," Journal of Dentistry, vol. 29, no. 6, pp. 395-400, 2001.

[31] A. Azzopardi, D. W. Bartlett, T. F. Watson, and M. Sherriff, “The surface effects of erosion and abrasion on dentine with and without a protective layer," British Dental Journal, vol. 196, no. 6, pp. 351-354, 2004.

[32] P. A. Brunton, K. S. Kalsi, D. C. Watts, and N. H. F. Wilson, "Resistance of two dentin-bonding agents and a dentin densensitizer to acid erosion in vitro," Dental Materials, vol. 16, no. 5, pp. 351-355, 2000.
[33] G. Sundaram, R. Wilson, T. F. Watson, and D. Bartlett, “Clinical measurement of palatal tooth wear following coating by a resin sealing system," Operative Dentistry, vol. 32, no. 6, pp. 539-543, 2007.

[34] M. D. Lagerweij, W. Buchalla, S. Kohnke, K. Becker, Á. M. Lennon, and T. Attin, "Prevention of erosion and abrasion by a high fluoride concentration gel applied at high frequencies," Caries Research, vol. 40, no. 2, pp. 148-153, 2006.

[35] S. Oguz Ahmet, M. M. Mutluay, Z. Seyfioglu Polat, R. Seseogullari Dirihan, B. Bek, and A. Tezvergil-Mutluay, "Addition of benzalkonium chloride to self-adhesive resin-cements: some clinically relevant properties," Acta odontologica Scandinavica, vol. 72, no. 8, pp. 831-838, 2014.

[36] M. Schiltz-Taing, Y. Wang, B. Suh, D. Brown, and L. Chen, "Effect of tubular orientation on the dentin bond strength of acidic self-etch adhesives," Operative Dentistry, vol. 36, no. 1, pp. 86-91, 2011.

[37] J. B. Cruz, T. L. Lenzi, T. K. Tedesco, C. D. A. B. Guglielmi, and D. P. Raggio, "Eroded dentin does not jeopardize the bond strength of adhesive restorative materials," Brazilian Oral Research, vol. 26, no. 4, pp. 306-312, 2012.

[38] C. M. Machado, A. C. Zamuner, K. C. Modena, S. K. Ishikiriama, and L. Wang, "How erosive drinks and enzyme inhibitors impact bond strength to dentin," Brazilian Oral Research, vol. 29, no. 1, pp. 1-7, 2015.

[39] F. M. Collares, S. B. Rodrigues, V. C. B. Leitune, R. K. Celeste, F. B. De Araújo, and S. M. W. Samuel, "Chlorhexidine application in adhesive procedures: a meta-regression analysis," Journal of Adhesive Dentistry, vol. 15, no. 1, pp. 11-18, 2013.

[40] P. C. P. Komori, D. H. Pashley, L. Tjäderhane et al., "Effect of $2 \%$ chlorhexidine digluconate on the bond strength to normal versus caries-affected dentin," Operative Dentistry, vol. 34, no. 2, pp. 157-165, 2009.

[41] J. Kim, T. Uchiyama, M. Carrilho et al., "Chlorhexidine binding to mineralized versus demineralized dentin powder," Dental Materials, vol. 26, no. 8, pp. 771-778, 2010.

[42] V. Hass, I. V. Luque-Martinez, M. F. Gutierrez et al., "Collagen cross-linkers on dentin bonding: stability of the adhesive interfaces, degree of conversion of the adhesive, cytotoxicity and in situ MMP inhibition," Dental Materials, vol. 32, no. 6, pp. 732-741, 2016.

[43] R. Seseogullari-Dirihan, M. M. Mutluay, D. H. Pashley, and A. Tezvergil-Mutluay, "Is the inactivation of dentin proteases by crosslinkers reversible?” Dental Materials, vol. 33, no. 2, pp. e62e68, 2017. 

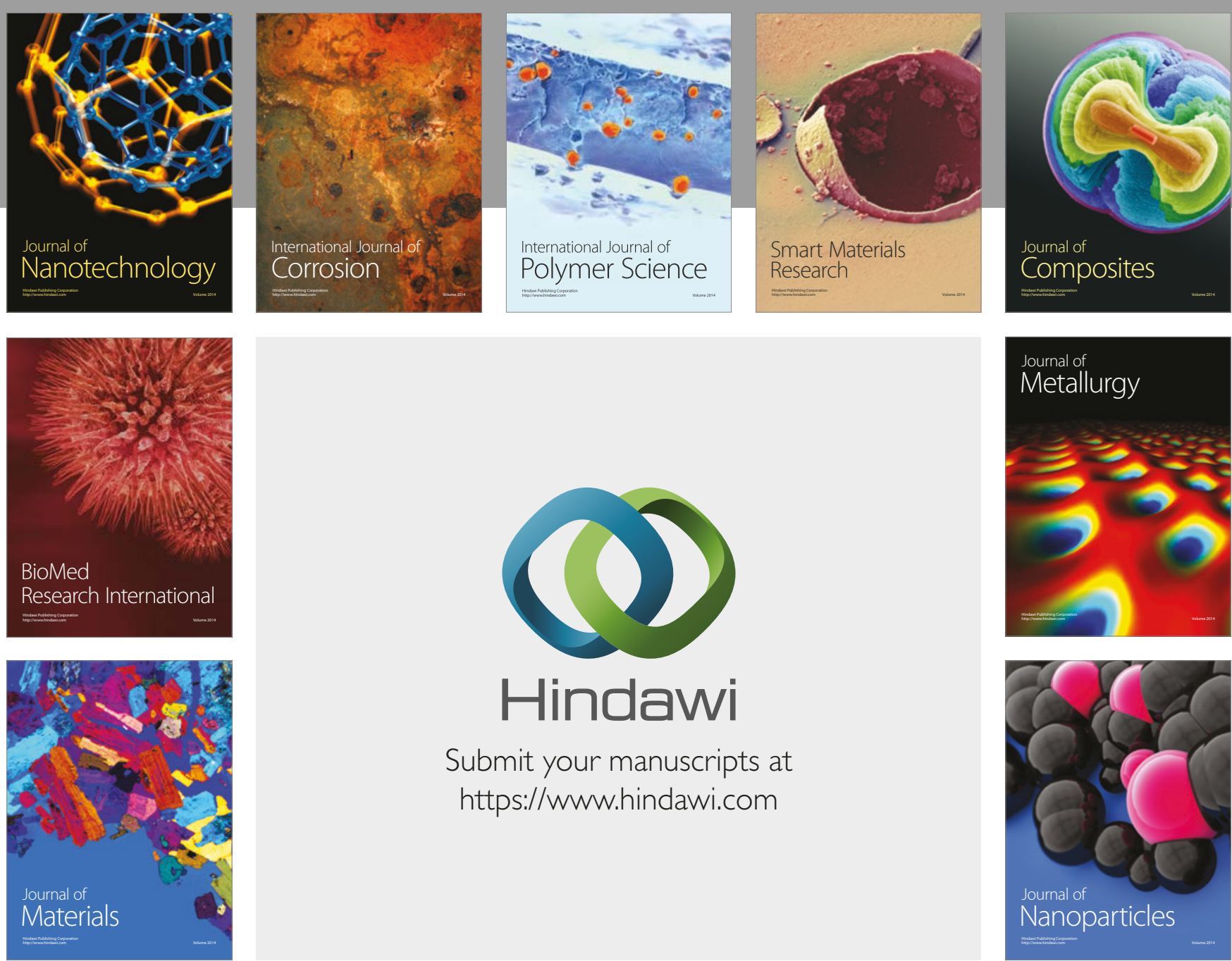

\section{Hindawi}

Submit your manuscripts at

https://www.hindawi.com
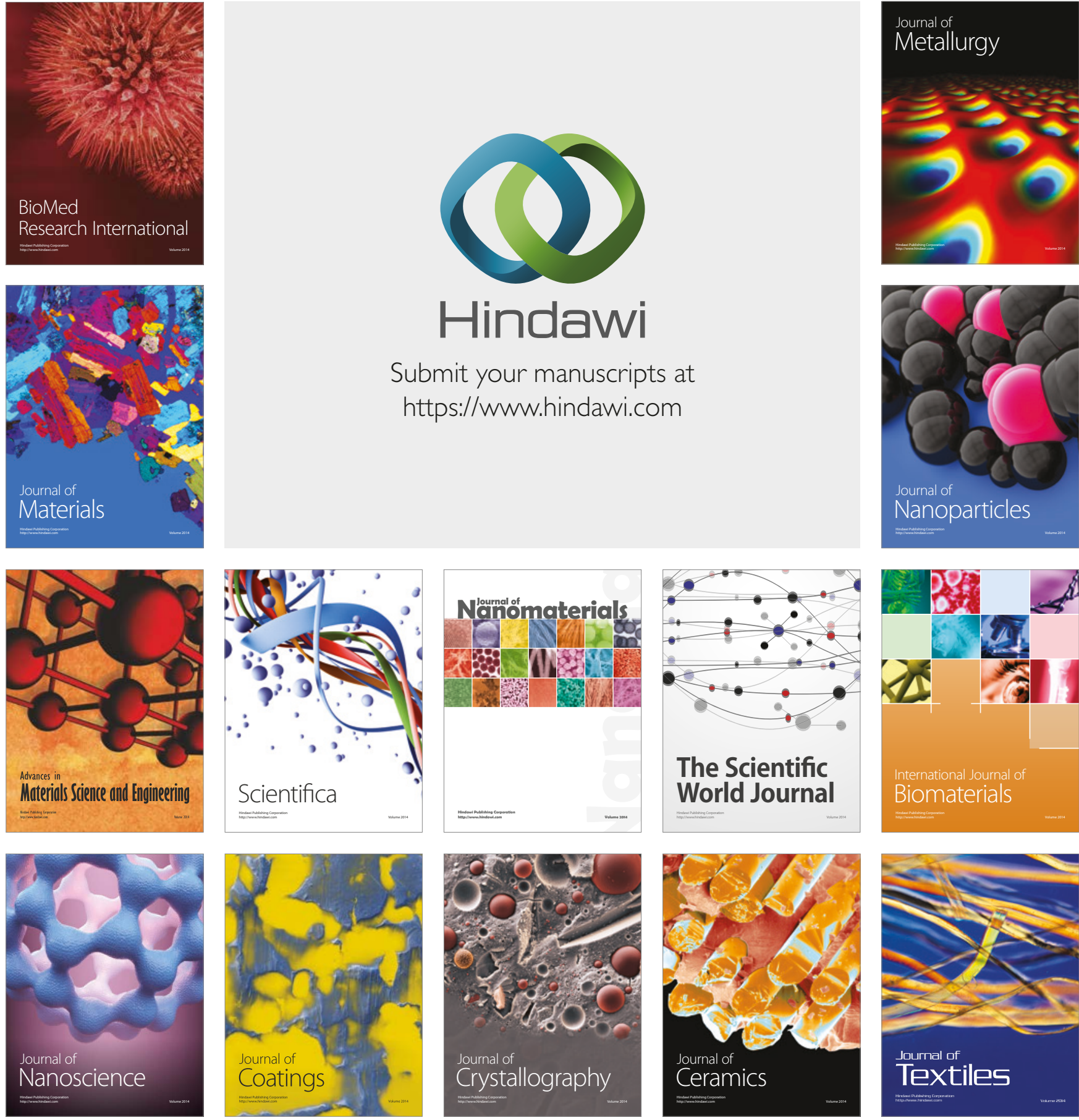

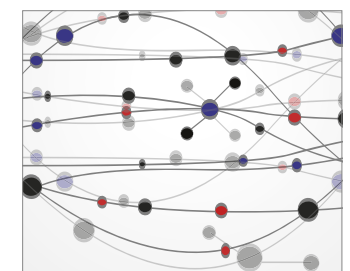

The Scientific World Journal
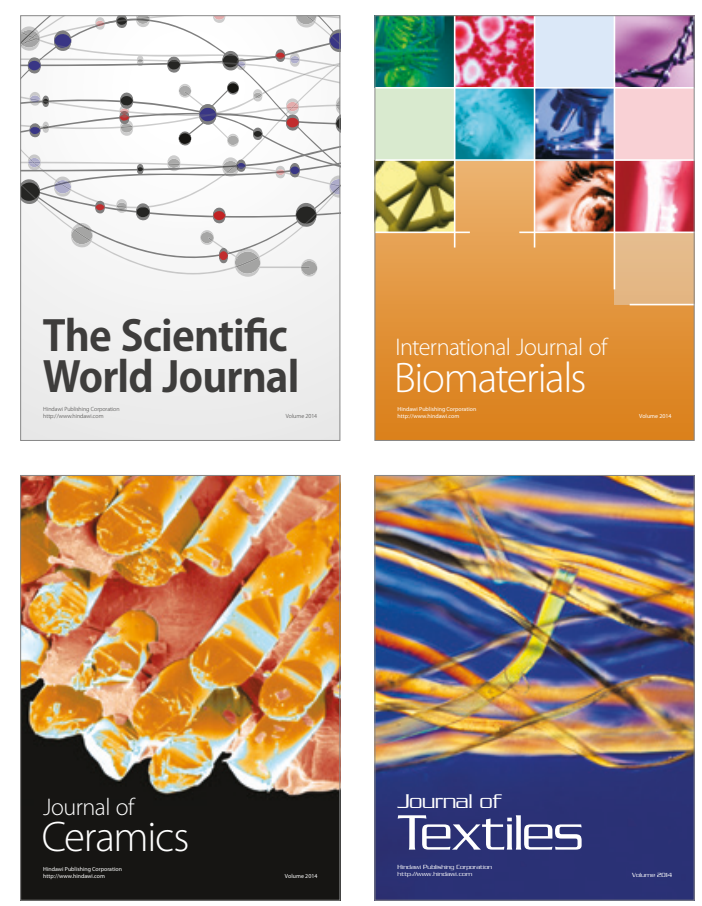